\title{
PREVALENCIA DE LESIONES EN PATINADORES DE LAS CATEGORÍAS MAYORES DEL CLUB TEQUENDAMA DE BOGOTÁ
}

Cindy Tatiana Sánchez Bonilla?

Laura Elizabeth Castro Jiménez²

\section{Presentación}

Las lesiones están inevitablemente relacionadas con la actividad física, el ejercicio físico y por su puesto con cualquier actividad deportiva, ya sea en el plano recreativo como competitivo. El objetivo de este estudio es analizar la prevalencia de las lesiones que se presentan en el patinaje de carreras, específicamente en 26 patinadores que se desempeñan como deportistas activos del Club Tequendama de Bogotá. Como metodología se planteó un estudio cuantitativo, en el que se aplicaron encuestas a los patinadores de las categorías mayores del club. Dentro de los resultados se encontró que en este deporte las lesiones más frecuentes son las fracturas y que por ende el tejido más afectado por lesiones es el tejido óseo, además, que el mecanismo más recurrente de lesión es el traumatismo directo dado generalmente por fuertes caídas. Además de lo anterior, se pudo observar que la zona del cuerpo más afectada por las lesiones sufridas por los patinadores es la zona del tren inferior.

1 Profesional de Cultura Física, Deporte y Recreación, Semillero de investigación GICAEDS línea de Rehabilitación Deportiva, Universidad Santo Tomás. Correo electrónico: cindysanchez@usantotomas.edu.co

2Facultad de Cultura física deporte y recreación, Grupo de investigación GICAEDS, Bogotá-Colombia. 
A partir de la presente investigación se busca hacer un acercamiento al nivel de prevalencia de las lesiones en el deporte del patinaje de carreras. Las lesiones han sido y siempre van a ser un aspecto inherente a la práctica deportiva; es decir, que aun conociendo los innumerables beneficios que resultan de realizar cualquier tipo de actividad física o deportiva, es ineludible evidenciar que estas prácticas también cuentan con ciertas desventajas y una de ellas, es la aparición de las lesiones.

Muchas pueden ser las causas que contribuyan a que se genere cualquier tipo de lesión; la mala ejecución de determinados movimientos o el sobreuso de algunos grupos musculares son algunas de las muchas causas que pueden contribuir a que aparezcan, las que se conocen como, lesiones deportivas. Con este estudio se quiere llegar a conocer para el caso específico del patinaje de carreras, cuáles son las lesiones que prevalecen en este deporte y las causas que lleven a la limitación de la práctica deportiva. Además de lo planteado anteriormente, se debe tener en cuenta que en el patinaje de carreras se cuenta con un alto riesgo de accidentalidad que puede llegar a incidir considerablemente en el aumento de la aparición de lesiones en este deporte.

Se hace necesario conocer los datos de prevalencia de los diferentes tipos de lesiones deportivas que se presentan en la práctica del patinaje de carreras para que se pueda aplicar un proceso de prevención de las mismas desde una etapa más temprana, tanto por parte del cuerpo técnico como de los mismos deportistas. Tener una idea clara de las lesiones más comunes en este deporte ayuda a que ese proceso preventivo sea pertinente, de mayor eficacia y tal vez ayude a la reducción en la aparición de las lesiones deportivas, por lo menos de las que se puede tener algún tipo de control, porque como se aclaró, este deporte cuenta con un riesgo alto de accidentalidad durante su práctica. 


\section{Escenarios de la formación investigativa}

\section{|Marco Teóricol}

Se hace pertinente profundizar en los conceptos de lesión deportiva y tipos de lesiones, para que así se pueda lograr la adecuada comprensión del fenómeno a estudiar en esta investigación. En primera instancia, se debe tener determinada noción de lo que es la lesión antes de poder hablar de lesión deportiva. La lesión, vista desde el punto de vista sanitario o medicinal, es la alteración de las condiciones normales del mantenimiento de un cuerpo o un organismo; Romo (2000) la define como toda alteración anatómica o funcional que puede ser ocasionada por uno o más agentes externos o internos; es decir que hay lesión cuando las características normales de un cuerpo u organismo se ven alteradas por factores ya sean internos (microorganismos) o externos (golpes). A partir de lo anterior, se puede determinar que son los factores ocasionados durante una práctica deportiva en específico, lo que hace que exista una lesión deportiva, sería entonces, el daño tisular (de los tejidos del organismo) que se produce como consecuencia de la participación en algún deporte o ejercicio físico (Bahr y Maehlum, 2007); tal y como lo señalan Osorio, Clavijo, Arango, Patiño y Gallego (2007), la lesión deportiva se entiende como "la que ocurre cuando los atletas están expuestos a la práctica del deporte y se produce alteración o daño en un tejido, afectando el funcionamiento de la estructura".

Por otro lado, Pfeiffer y Mangus (2007) afirman que no existe una definición "única y universal" de lo que es la lesión deportiva, y plantean tres características generales para que una lesión sea clasificada como lesión deportiva:

1. Ser producto de la participación en un entrenamiento o partido.

2. Exigir atención médica por parte del preparador físico o el médico del equipo.

3. Causar una restricción en la participación o rendimiento del deportista durante uno o más días con posterioridad al día de la lesión.

De lo anterior se puede destacar que las lesiones deportivas son las que se adquieren durante la práctica de cualquier deporte y que ocurren en algunos de los tejidos que componen el organismo humano: óseo, muscular, ligamentario y tendinoso. Así mismo, se plantea una "clasificación médica" que establece a 
la lesión en dos categorías principales (Pfeiffer y Mangus, 2007): Las lesiones agudas y las lesiones crónicas. En cuanto a las primeras, se puede especificar que son las lesiones que se caracterizan por una aparición o inicio repentino, por consecuencia de un hecho significativamente traumático y que además presentan cierta sintomatología tal como "dolor, inflamación y pérdida de la capacidad funcional", asimismo se identifican por tener una duración menor a un mes. Por otro lado, las lesiones crónicas son las que se caracterizan por un inicio lento y que implican el daño gradual de la estructura afectada, es decir, que se desarrollan progresivamente y no dependen de ningún evento traumático en particular, sino que se asocian a deportes que requieren movimientos altamente repetitivos y continuos; estas lesiones se caracterizan porque presentan una duración mayor a tres meses.

Bahr y Maehlum (2007) también clasifican las lesiones en dos grupos según el mecanismo de lesión de las mismas y el comienzo de sus síntomas, estos dos grupos son: lesiones directas y lesiones indirectas o por uso excesivo. Las lesiones directas, al igual que la anterior definición, son las que ocurren de manera repentina y se originan a partir de una causa claramente definida, (actividades deportivas que puedan conllevar al riesgo de fuertes caídas o deportes que requieran de contacto físico muy fuerte y frecuente por parte de los deportistas). Por el contrario, las lesiones por uso excesivo o indirectas son las que ocurren o se desarrollan de forma gradual, y los autores señalan que estas son predominantes en deportes aeróbicos donde se presentan sesiones de entrenamiento monótonas y muy prolongadas, o en deportes que requieren de movimientos altamente repetitivos.

Ahora se hace pertinente hablar de otra clasificación de lesiones deportivas, enfocada hacia el tipo de tejido que afectan: Lesiones musculares, lesiones ligamentarias, lesiones tendinosas y lesiones óseas.

a. Las lesiones ligamentarias aparecen cuando hay ruptura de los ligamentos y estas ocurren generalmente por traumatismos agudos, es decir, "el mecanismo más común de lesión consiste en una sobrecarga repentina con distención del ligamento mientras la articulación se encuentra en una posición extrema” (Bahr y Maehlum, 2007). Los esguinces son las lesiones de los ligamentos y se dividen en tres tipos según su gravedad: De primer grado, que son los esguinces de menos gravedad ya que únicamente incluyen una baja discapacidad funcional, dolor leve y presentan daños menores de los ligamentos. El esguince de segundo grado presenta mayor daño en los ligamentos y por ende más dolor e inflamación, además de movilidad anormal del segmento lesionado. Por último, el esguince más grave, es el esguince de tercer grado donde se evidencia la ruptura total de los ligamentos, es decir, mayor daño, mayor dolor y mayor inflamación con relación a los dos primeros grados de esguince (Pfeiffer y Mangus, 2007). 


\section{Escenarios de la formación investigativa}

Las rupturas ligamentarias pueden producirse en el interior de la sustancia ligamentosa o en el sitio de la unión del hueso con el ligamento, aunque también puede presentarse una "fractura por avulsión" que es cuando el ligamento arranca una porción de hueso.

b. Las lesiones tendinosas son rupturas del tejido conjuntivo que une el hueso con el músculo y pueden ser de tipo agudo (repentinas) o de tipo crónico o por uso excesivo (progresivas). Estas se producen cuando la fuerza aplicada excede la tolerancia del tendón, pueden ser parciales o totales, $y$ al igual que los ligamentos se pueden dar en el medio de la sustancia del tejido, en la unión osteotendinosa o por avulsión. Bahr y Maehlum (2007) señalan los diferentes términos para describir los diferentes tipos de afecciones que se dan en los tendones:

Tendinitis: inflamación del tendón.

Tenosinovitis: inflamación de la vaina tendinosa.

Tenoperiostitis: inflamación de las inserciones tendinosas.

Periostitis: inflamación del periostio.

Bursitis/Hemobursitis: inflamación de la bursa, con probable hemorragia asociada.

c. Por otra parte, las fracturas y las luxaciones hacen parte de las lesiones que afectan al tejido óseo y además a las articulaciones. En cuanto a las fracturas se pueden reconocer dos clases, las primeras son las fracturas cerradas, donde el hueso no atraviesa la piel, es decir, que no hay contacto de la parte interna del cuerpo con la parte externa (medio ambiente). Las segundas son las fracturas compuestas o abiertas, que son fracturas que a diferencia de las cerradas sí presentan contacto de la parte externa con la interna porque hay extremos del hueso sobresaliendo por la piel, presentando una herida abierta (Pfeiffer y Mangus, 2007). Entre los tipos de fractura se pueden encontrar las de tallo en verde, transversas, oblicuas y conminutas.

En cuanto a las luxaciones, estas se definen como "el desplazamiento de las superficies contiguas de los huesos que forman una articulación" (Pfeiffer y Mangus, 2007). Según la gravedad de la lesión se puede dividir en dos tipos: Subluxación (cuando hay desplazamiento parcial de los huesos de la articulación) y luxación (cuando los huesos de la articulación se desplazan completamente).

d. Una de las lesiones musculares son las distenciones y al igual que los esguinces, estas se clasifican según su gravedad. Las de primer grado son las más leves e implican poco daño en la estructura muscular y tendinosa, así mismo presentan dolor fuerte, inflamación y en ocasiones espasmos musculares. En las distenciones musculares de segundo grado, además de 
presentarse mayor dolor e inflamación que en la de primer grado, también presenta una perdida funcional moderada y un daño mayor del tejido. Por último en las distenciones de tercer grado se evidencia una ruptura completa en la estructura de los tejidos, ya sea en la unión del tendón, en los tejidos entre el tendón y el músculo (unión musculo tendinosa) o en los tejidos del músculo mismo. Esta lesión implica una perdida funcional importante. (Pfeiffer y Mangus, 2007).

Otra de las lesiones que se presentan en los músculos son las contusiones, estas son las que se producen por golpes en la superficie del cuerpo que comprimen los tejidos subcutáneos y la piel. Son lesiones que se caracterizan por dolor, rigidez, hinchazón, equimosis y hematomas (Pfeiffer y Mangus, 2007). Las contusiones son consideradas normalmente como lesiones menores, pero pueden agravarse si no se tratan correctamente, ya que podría acarrear formaciones osiformes en el tejido muscular. Por otra parte, el desgarro o laceraciones musculares (Bahr y Maehlum, 2007), otra de las posibles lesiones que pueden presentar los músculos, es la perdida de continuidad de las fibras musculares y su gravedad se ve determinada a partir del número de fibras que se ven afectadas en el momento de la lesión (Hinrichs, 1995). Para finalizar con las lesiones que afectan el tejido muscular, se quiere hablar de la contractura. Esta es una contracción fisiológica que es consecuencia de una situación dolorosa y que tiene un inicio brusco (Prieto, 1998), por otro lado, López y López (2008) definen la contractura como un acortamiento doloroso e involuntario de un músculo.

Otro de los tejidos que puede verse afectado por las lesiones deportivas es el tegumentario. Este está compuesto por la piel y recubre la totalidad de la superficie corporal humana (Daza, 2007) y como los tejidos anteriormente presentados, este también está expuesto a sufrir lesiones por la práctica deportiva. Las lesiones cutáneas pueden implicar limitación de la práctica del deporte (según la gravedad) y la eventual formación de cicatrices; se pueden clasificar en heridas cerradas y en heridas abiertas, las cuales las primeras se pueden dar por contusiones o aplastamientos y las segundas suceden cuando existe perdida de continuidad en la piel o en las mucosas. Estas lesiones pueden llegar a ser de gran cuidado, porque así como hay lesiones cutáneas que permiten la continuación de la práctica deportiva, hay otras que requieren de atención porque pueden presentar complicaciones que conlleven a infecciones de la piel o de los órganos situados debajo de ella (Hinrichs, 1995).

Otro de los temas pertinentes para el desarrollo de esta investigación es el que hace referencia al deporte en el que se va a aplicar este estudio, el patinaje de carreras. Este deporte, también conocido como patinaje de velocidad, es una de las modalidades del patinaje que se practica en pista o en ruta, con patines de línea (Grelon, 1998) que se componen de la bota, el chasis y las ruedas y que con el paso del tiempo han ido evolucionando. Pista se le llama al recorrido de 
competencia que se puede encontrar en un espacio abierto (al aire libre) o en una instalación cubierta; debe medir 200 metros de largo y 6 metros de ancho. Esta pista debe contar con dos rectas del mismo largo y con dos curvas totalmente simétricas, según las medidas estipuladas por el Comité Internacional de Carreras (CIC) nombradas anteriormente. A diferencia de la pista, la ruta consta de un recorrido asimétrico, cuenta con unas medidas diferentes, de largo no puede superar los 600 metros ni disminuir de los 400 metros y el ancho nunca puede ser inferior a 5 metros. Para este deporte, los atletas se especializan en pruebas de velocidad (que se disputan individualmente o por equipos) o en pruebas de fondo, según las capacidades físicas de cada uno; las distancias de las pruebas varían de 200 metros hasta la maratón de 42 kilómetros, pasando por los 300, 500, 1.000, 3.000, 5.000, 10.000, 15.000 y 20.000 metros (Federación Colombiana de Patinaje, 2005). 


\section{Análisis de resultados}

Con la aplicación de las encuestas a los deportistas del Club Tequendama y la ayuda del software de análisis estadístico descriptivo SPSS versión 21, se obtuvieron los resultados expuestos a continuación.

\section{Descripción de los resultados obtenidos con relación a los datos socio-demográficos de los deportistas:}

Para empezar con la información que hace referencia a los datos socio-demográficos de los patinadores, se quiere describir en primera instancia los datos obtenidos con lo relacionado al género de las personas encuestadas. A partir de los resultados, se pudo evidenciar que el 73,1\% de las personas a las que se les aplicó la encuesta son mujeres y el 26,9 \% restante son hombres (tabla 2), evidenciándose la moda en el género femenino (tabla 1). En cuanto a las edades de los deportistas encuestados (tabla 3), éstas oscilan entre los 14 y los 32 años, mostrando una mayor frecuencia en las edades de 15 y 16 años, cada uno con un porcentaje de $23,1 \%$. La media y la moda arrojadas por el software estadístico son 17,27 y 15 años respectivamente (tabla 1 ).

Por otra parte, en lo que se refiere al estrato socio-económico, los resultados permitieron conocer que los deportistas encuestados pertenecen a los estratos 2,3 y 4, habiendo una frecuencia del $65,4 \%$ en el estrato 3 . Se puede evidenciar entonces, en la tabla 4, que los estratos 1, 5 y 6 no contaron con ninguna frecuencia de datos de acuerdo a las respuestas obtenidas en las encuestas, es decir, que ninguno de los deportistas a los que se les aplicó la encuesta pertenece a alguno de estos estratos.

Para el caso de la modalidad en la que se desempeñan los patinadores encuestados (figura 1), el 3,8 \% de la población testificó no participar en una modalidad determinada; la mayoría de personas, representadas con el 84,6 \%, expresaron desempeñarse en la modalidad de fondo, es decir, en pruebas que oscilan en distancias entre los 10.000 y los 20.000 metros (Federación Colombiana de Patinaje, 2005). Mientras que el 11,5\% de los encuestados dijeron que se desempeñan como velocistas en la práctica del patinaje, compitiendo así en pruebas 
contrarreloj de 200 y 300 metros y en sprints de 500 y 1.000 metros (Federación Colombiana de Patinaje, 2005). Por otra parte, en lo que se puede observar en cuanto a la inscripción y participación en los entes reguladores del deporte a nivel distrital y nacional (Liga de Patinaje de Bogotá y Federación Colombiana de Patinaje), el 88,5\% de los deportistas que respondieron a la encuesta afirmaron estar inscritos a estas dos entidades y el 11,5\% restante especificaron no estar afiliados ni a la liga ni a la federación; lo que quiere decir que estos deportistas no pueden participar en ningún campeonato oficial que organicen y avalen estas dos entidades a nivel distrital o nacional.

Para dar termino con los resultados obtenidos sobre la información referente a los datos socio-demográficos de los deportistas del club Tequendama, se puede evidenciar en la tabla 5 que el 46,2\% de los encuestados pertenecen a la categoría juvenil, es decir que son 13 deportistas los que afirmaron tener entre 15 y 16 años. La segunda categoría con mayor cantidad de deportistas, es la de mayores (deportistas con edades de 17 años en adelante) con un porcentaje de 38,5 \% y el 15,4 \% restante de los encuestados pertenecen a la categoría pre juvenil, señalando que solo 4 deportistas afirmaron tener 14 años en la actualidad.

\section{Descripción de los resultados obtenidos con relación a los datos sobre la práctica del patinaje de carreras:}

A partir de los interrogantes que se plantearon en la encuesta para lograr información detallada sobre la práctica del deporte se obtuvieron los siguientes datos: En cuanto a la pregunta que hacía referencia al tiempo de práctica del patinaje de forma competitiva, es decir, el tiempo que se llevara participando en campeonatos distritales y nacionales, las encuestas arrojaron que el 96,2\% de los deportistas respondieron que llevaban compitiendo durante 5 años o más y el 3,8 \% restante respondió que llevan de 3 a 4 años participando de forma activa en campeonatos a nivel distrital y nacional (figura 2). Por otra parte, se pudo observar con los resultados que ninguno de los deportistas encuestados respondió que llevan compitiendo de 1 a 2 años. Es importante resaltar que haciendo una relación de los datos obtenidos con esta interrogante y con las respuestas dadas por los deportistas en cuanto a la pregunta que hacia relación a la inscripción y participación en los entes reguladores del deporte en Bogotá y a nivel nacional, se puede establecer que existe una incongruencia en las respuestas, ya que si los deportistas no se encuentran inscritos a la liga y a la federación, estos no podrán participar de los campeonatos oficiales avalados por estos entes; es decir, que los deportistas que respondieron no estar afiliados tanto a la liga como a la federación, no pudieron haber respondido a la pregunta de cuánto tiempo llevaban practicando el deporte competitivamente. Al momento de detectar esta inconsistencia, durante la aplicación de la encuesta, se recurrió a preguntarles el porqué de su respuesta y las tres personas que habían afirmado no estar inscritos en los entes coincidieron en responder que durante algún tiempo atrás compitieron y 
que actualmente no lo hacían, una de esas personas afirmó no querer competir nuevamente y relataba que practicaba el deporte como medio de distracción y las otras dos personas afirmaron querer retomar las competencias nuevamente.

En referencia al interrogante que pretendía conocer la cantidad de horas que entrenan al día, se obtuvieron los siguientes resultados: el 11,5\% manifestaron entrenar de 6 a 7 horas en el día, el 42,3\% de los encuestados respondieron que entrenan de 2 a 3 horas y el 46,2 \% restante afirmó que la duración de su entrenamiento en el día es de 4 a 5 horas en la actualidad, haciendo de este último porcentaje la moda, es decir el valor con mayor frecuencia de datos como se observa en la tabla 6 .

Para la pregunta que hace referencia a la cantidad de días que entrenan a lo largo de la semana, se puede observar que el $100 \%$ se distribuye en dos respuestas de las cuatro posibles. El menor valor, que corresponde a un 46,2 \%, refleja que 12 de los 26 deportistas encuestados afirman entrenar de 5 a 6 días de los siete días de la semana, y por otra parte, el 53,8\% restante testifica entrenar 7 días a la semana (figura 3). En cuanto a las otras dos posibles respuestas, no hay ningún patinador que afirme entrenar con una frecuencia menor a 4 días, ya que los valores de 1 a 2 días y 3 a 4 días no obtuvieron ninguna respuesta.

Para la pregunta que pretendía conocer sobre la cantidad de sesiones de entrenamiento que realizan los deportistas a lo largo de la semana se obtuvieron datos en las cuatro respuestas posibles. La respuesta que mayor frecuencia presentó fue la que señalaba de 5 a 7 sesiones a la semana con un valor del $57,7 \%$, las respuestas de 11 a 13 sesiones a la semana, 14 sesiones o más y 8 a 10 sesiones tuvieron valores de $26,9 \%, 11,5 \%$ y $3,8 \%$ respectivamente como se puede observar en la figura 4.

En cuanto a las opiniones que tienen los deportistas sobre las condiciones de la pista, la moda para el caso de esta pregunta fue la respuesta de que sí son óptimas las condiciones del lugar donde se practica el patinaje de carreras con un valor del $57,7 \%$; mientras que el $42,3 \%$ de los deportistas encuestados respondieron que las condiciones de la pista no son las adecuadas (tabla 7).

Para finalizar con los datos obtenidos referentes a la práctica deportiva, se quiso saber cuáles elementos de protección eran los que los deportistas usaban durante la práctica del patinaje. Los resultados arrojados por las encuestas muestran que el $65,4 \%$ de los deportistas usan casco y guantes. El 15,4 \% usa casco y algún otro elemento de protección; el 11,5 \% afirma usar casco, guantes y otros; y por último, el 7,7\% restante aseguró que para la práctica deportiva únicamente utilizan casco como protección (figura 5). Para conocer que otros implementos de protección utilizaban, mientras se aplicó la encuesta, se les pregunto a los deportistas que contaban usar otros elementos de protección, cuáles eran esos elemen- 
tos y a partir de eso se pudo conocer que los antifracturantes, el anillo rotuliano y las rodilleras son utilizados como fin para la protección o avance de lesiones.

\section{Descripción de los resultados obtenidos con relación a la pre- valencia de lesiones deportivas en el patinaje de carreras:}

Para empezar a conocer la prevalencia que hay de lesiones deportivas en este deporte en específico, se quiso saber cuántos de los encuestados han sufrido alguna vez durante la práctica deportiva cualquier tipo de lesión. Para esta pregunta la moda fue que sí han sufrido alguna lesión por la práctica del patinaje. Como se puede observar en la figura 6 , el valor de los que respondieron afirmativamente a la pregunta fue de un 80,8 \% y el 19,2\% restante afirmó nunca haber sufrido una lesión por la práctica del patinaje; lo que de aquí en adelante representará datos perdidos, por ende solo se hablará del porcentaje que respondió afirmativamente a la pregunta. A partir de lo anterior, del total de las personas que afirmaron haber vivenciado alguna lesión, el 46,2 \% respondió que en lo largo de su vida deportiva han sufrido de 1 a 2 lesiones, el 23,1\% respondió haber sufrido de 3 a 4 lesiones y por último, para completar el $80,8 \%$ de los que sí han sufrido lesiones, el 11,5\% restante contestó que han sufrido 5 lesiones o más a lo largo de su vida deportiva (tabla 8).

En cuanto a la cantidad de lesiones que han tenido en el último año, ese 80,8 \% se distribuye en los siguientes valores: el 46,2\% respondió que han tenido de 1 a 2 lesiones, el 30,8\% afirma no haber sufrido ninguna lesión en el último año y el último 3,8\% responde a la pregunta diciendo que han tenido 5 lesiones o más en el transcurso del año. Por último y para conocer el periodo más frecuente en el que se presentaron estas lesiones en este deporte, los encuestados respondieron que es en el entrenamiento con un $69,2 \%$, el periodo en el que creen se han presentado con mayor frecuencia las lesiones que han tenido. El 11,5\% restante respondió que es en el periodo competitivo en el que creen que se presentan las lesiones y el periodo postcompetitivo no tuvo ninguna frecuencia en las respuestas por parte de los encuestados.

Descripción de los resultados obtenidos con relación a las lesiones más recientes:

Para empezar a conocer todo lo pertinente en cuanto a las lesiones presentadas por los deportistas del club Tequendama de Bogotá, la pregunta hace referencia a la fecha en que estas se presentaron. Del total de los encuestados, siete personas (el 26,9\%) respondieron que la lesión más reciente la sufrieron hace un mes o menos, lo que puede significar que los deportistas se encuentran en pleno proceso de rehabilitación y recuperación. Otro 26,9 \% de los deportistas encuestados afirmó haber sufrido su última lesión hace un año o más y el último $26,9 \%$ respondió a la interrogante diciendo que la última lesión la sufrió hace 2 a 6 meses. 
Ahora, del $100 \%$ de las personas que dijeron haber tenido una lesión (sin contar los datos de los que no han sufrido alguna lesión por la práctica del deporte), el $42,9 \%$ afirmaron que el tejido óseo es el más afectado por las lesiones que han sufrido recientemente. Como se puede observar en la figura 7, el tejido muscular, el tendinoso, el ligamentario y el tegumentario también fueron afectados por las últimas lesiones sufridas por los patinadores. Para este caso, ningún deportista presentó alguna lesión que presentara algún compromiso a nivel articular.

En cuanto al tipo de lesión presentada, se puede observar en la figura 8, que con un $33,3 \%$ la fractura presenta mayor frecuencia en las respuestas dadas por los deportistas.Después de esta lesión ósea, es la contractura muscular la segunda lesión más frecuente con un $19 \%$, seguido del esguince con un 14,3\%. Otras lesiones que manifiestan los deportistas haber sufrido recientemente son tendinitis, bursitis y fisuras; y por último, la lesión menos frecuente es la herida abierta con un $4,8 \%$.

En cuanto al mecanismo con el que se presentaron las lesiones más recientes que sufrieron los patinadores encuestados, la respuesta que desplegó un mayor número de frecuencias fue el traumatismo directo con un $66,7 \%$ frente a un 33,3 $\%$ de deportistas que afirmaron que su lesión tuvo un desarrollo progresivo. Por otra parte, en lo que hace referencia al tipo de lesión, según la "clasificación médica", se pudo observar a partir de los resultados que el 66,7 \% de los deportistas afirmaron haber sufrido lesiones repentinas y por algún hecho traumático como caídas o golpes. El 33,3 \% restante afirmo haber tenido lesiones de tipo crónico.

Con la información obtenida sobre las zonas del cuerpo afectadas por las lesiones, se pudo determinar que la mayor frecuencia de datos se presentó en las extremidades inferiores con un valor de $57,1 \%$, seguido de las extremidades superiores con un $28,6 \%$ y la zona del cuerpo que presentó menor cantidad de lesiones fue el tronco con un 14, 3 \% (tabla 9). Para el caso de las lesiones recientes, los deportistas no reportaron haber sufrido ningún tipo de lesión en la zona de la cabeza.

Para la pregunta que se formuló para conocer si a los deportistas que presentaron lesión se les prescribió algún tipo de incapacidad, se obtuvieron los siguientes datos: el 66,7\% de los deportistas respondieron que sí se les fue formulada incapacidad médica, mientras que el 33,3 \% restante afirmó que no se le formuló ninguna incapacidad. De los deportistas a los que se les formulo incapacidad, el $64,3 \%$ respondió que la incapacidad que se les formulo fue por un lapso de tiempo de 1 mes o menos; el 21,4\% manifestó que el tiempo de incapacidad fue por un tiempo de 2 a 6 meses y por último, el 14,3\% de los encuestados respondió que la incapacidad fue por un año o superior a esto (figura 9). También se quiso saber si los deportistas cumplieron el tiempo de incapacidad o si por el contrario decidieron continuar con la práctica deportiva. Para el interrogante 
sobre si se cumplió o no el tiempo sugerido en la incapacidad, se descubrió que el $57,1 \%$ de los que fueron incapacitados afirmaron no haber cumplido con esa incapacidad, mientras que el 42,9\% restante aseguró que sí se cumplió con el tiempo de incapacidad médica.

A los deportistas también se les preguntó, ya sea que hayan tenido incapacidad o no, cuánto tiempo esperaron para reanudar la práctica del patinaje. Un 42,9\% de los encuestados que han sufrido alguna lesión aseguró que el tiempo en que demoraron en retomar su práctica deportiva fue de 1 mes o menos, otro 38,1\% afirmó no haber suspendido el entrenamiento por la última lesión que sufrieron; el 9,5\% de los deportistas que presentaron lesión respondieron que duraron de 2 a 6 meses en retomar sus actividades deportivas y por último un 4,8\% y otro $4,8 \%$ respondieron haberse demorado de 7 a 11 meses y un año o más respectivamente (figura 10).

De la lesión más reciente que hayan tenido los patinadores, también se quiso saber si estas se trataron con algún tipo de rehabilitación. El 57,1 \% aseguró haber asistido a sesiones de rehabilitación que les ayudaran con sus lesiones deportivas, mientras que el otro $42,9 \%$ dijo que no vivenciaron ningún tipo de rehabilitación. De los que contaron haber asistido a rehabilitación, el 83,3 \% afirmó haber recibido diferentes tipos de tratamiento de los que se les expusieron en la encuesta (termoterapia, crioterapia, masoterapia, electroterapia, etc.) y solo el 16,7\% respondió a esta pregunta diciendo que el único tipo de rehabilitación a la que estuvieron expuestos fue al ejercicio terapéutico (ejercicios de fortalecimiento con thera-band, bases inestables, entre otras cosas). Para finalizar con lo referente a la rehabilitación, se quiso conocer si el proceso se finalizó o no; de estos datos se obtuvieron resultados del $58,3 \%$ en que los encuestados lesionados dijeron haber concluido su rehabilitación, frente a un $16,7 \%$ que dijo no haber terminado el proceso y un $25 \%$ que describió estar en la actualidad en procedimientos de rehabilitación para la recuperación de su lesión deportiva.

\section{Descripción de los resultados obtenidos con relación a las lesiones más graves:}

Para el caso de la lesión más grave, se inició también con la pregunta que pretendía conocer el tiempo en que sucedió la misma. La frecuencia (cantidad de respuestas) en este caso fue menor en comparación a las que hubo en la lesión más reciente; ya sea porque los deportistas han sufrido solo una lesión en toda su vida deportiva (que sería la lesión más reciente) o porque la lesión que expusieron como la más reciente, también fue la lesión más grave que hayan tenido. Para esta pregunta, el 42,3\% de los encuestados respondió que la tuvo hace 12 meses o más y un 3,8\% aseguró haber sufrido la lesión más grave hace 7 a 11 meses. Como se puede observar en la tabla 10 , la sumatoria de los porcentajes da $46,2 \%$ que representaría el total de las personas que hablaron sobre una se- 
gunda lesión. Es decir, que del 100 \% de los que habían respondido que en algún momento de su vida deportiva han sufrido alguna lesión, el 53,8 \% no dieron información sino de la primera lesión (lesión más reciente, o en algunos casos, la lesión más reciente también fue la más grave). Lo que quiere decir que de aquí en adelante, se tomará los datos de las 12 personas que hablaron sobre la segunda lesión como $100 \%$.

Frente al tipo de tejido más afectado por las lesiones, los datos arrojaron que el tejido óseo fue el que más respuestas obtuvo, es decir, que con un $50 \%$ es el tejido más afectado por las lesiones graves de los patinadores. El tejido muscular, es el segundo tejido más afectado con un $25 \%$ y el tejido tendinoso, el tegumentario y otro (respuesta para los que no se clasificaban en los tejidos dados como posibilidad de respuesta) lograron un $8,3 \%$ cada uno. Se puede observar entonces, que para esta pregunta, no se obtuvieron respuestas de que hayan afirmado que los tejidos ligamentario y articular hayan sido afectados por alguna lesión.

Para continuar, se quiso saber cuáles fueron esas lesiones que los patinadores reportaron como las más graves. Con el mayor porcentaje (50\%), las fracturas fueron las lesiones que los deportistas más han sufrido, seguido de las contracturas musculares con un $25 \%$ y con un $8,3 \%$ cada una, la bursitis, la herida abierta y otra lesión (clasificación diferente a la que se daba como posibilidades de respuesta). En este caso se puede observar que los porcentajes son exactamente iguales a la pregunta sobre el tejido afectado, pero analizando la información obtenida con las encuestas, se pudo determinar que no todas las fracturas reportadas afectaron el tejido óseo. Se presenta un caso en el que un deportista afirma haber sufrido de fractura en los dientes, que para el caso del tejido afectado se clasifico como otro ya que estos no catalogan como parte del tejido óseo. En cuanto a la lesión que se reporta como otra, es de una deportista que contaba que su lesión fue una desviación del coxis, lesión que se clasificó como que afecta el tejido óseo pero que no se podía catalogar dentro de las respuestas dadas sobre lesiones óseas (fractura y fisura).

A cerca del tipo de traumatismo que dio lugar a las lesiones más graves, el 66,7 $\%$ de los deportistas respondieron haber adquirido la lesión por un traumatismo directo, es decir por alguna causa claramente definida, como un golpe o un mal movimiento; y un 33,3\% respondió que sus lesiones se fueron desarrollando de forma gradual, es decir, fue un traumatismo indirecto el que incidió en la aparición de sus lesiones. En cuanto a la "clasificación médica", al igual que en las lesiones más recientes, los porcentajes de las respuestas son los mismos que el mecanismo de lesión. En este caso el 66,7 \% de los deportistas afirmaron haber sufrido lesiones que tuvieron duración menor a un mes, es decir, lesiones agudas; y el 33,3 \% restante aseguró haber tenido lesiones crónicas, lesiones que no dependieron de ningún hecho traumático en particular, sino que fueron creando molestias poco a poco que llevaron a la lesión. 


\section{Escenarios de la formación investigativa}

Para conocer otra característica importante de las lesiones graves reportadas por los deportistas, se preguntó cuál zona del cuerpo se vio afectada. Los datos arrojados por las encuestas a partir de esta pregunta mostraron que con un 50 $\%$, las extremidades inferiores son las regiones del cuerpo más afectadas por las lesiones graves que tuvieron los patinadores. La segunda zona más afectada es la de las extremidades superiores y por último, las respuestas de la zona del tronco y la de cabeza obtuvieron una frecuencia de una respuesta, es decir, un 8,3\% cada una (figura 12).

En cuanto a la información que se obtuvo sobre las incapacidades médicas que les fueron formuladas a los deportistas por la lesión más grave, un $75 \%$ de los deportistas respondió que sí tuvieron incapacidad, mientras que el $25 \%$ restante aseguró que no les fue prescrita incapacidad alguna. Así que, del total de deportistas a los que sí se les formuló, la respuesta a la pregunta sobre el tiempo de incapacidad fue: para el lapso de 2 a 6 meses el resultado fue de un $66,7 \%$, frente a un 22,2\% que respondió que la incapacidad duró 1 mes o menos; y un 11,1 \% restante al que se le formulo incapacidad por un lapso de 7 a 11 meses. Es decir que ninguno de los que se les formulo incapacidad, se les prescribió reposo por un periodo superior a 12 meses, ya que esta respuesta no contó con ninguna frecuencia (tabla 11).

Otra de las preguntas con referencia a la incapacidad médica, era relacionada a si esta se cumplió o no. De las respuestas dadas por los deportistas se obtuvieron los siguientes datos: un 44,4 \% refirió haber cumplido el tiempo de incapacidad que se les prescribió, mientras que el otro 55,6 \% negó haber cumplido la incapacidad formulada. A partir de esto, interesaba saber también el tiempo que se esperó para retomar la práctica del deporte. Del total de las personas que hablaron sobre una lesión grave, el 45,5\% dijo haber esperado de 2 a 6 meses para volver a entrenar, un 27,3\% de los deportistas respondió que retomó la práctica deportiva en 1 mes o menos y por último, otro $27,3 \%$ de los patinadores afirmó no haber suspendido las sesiones de entrenamiento por la lesión que presentaron (figura 13). A partir de los resultados arrojados por las encuestas, también se puede observar que ningún deportista respondió que se demoró en retomar su práctica deportiva más de 12 meses.

Para obtener información sobre la rehabilitación que hayan podido aplicar para la mejora de sus lesiones, se les preguntó a los deportistas si habían tenido algún tipo de rehabilitación y cuáles fueron esas rehabilitaciones a las que fueron sometidos. Para la primera de las preguntas los resultados que se obtuvieron fueron de un $75 \%$ contra un $25 \%$, de los cuales el mayor porcentaje corresponde a los deportistas que dijeron que sí se les hizo rehabilitación. De este total de deportistas, al 77,8\% se les aplicó diferentes tipos de rehabilitación (crioterapia, electroterapia, entre otros), mientras que el 22,2 \% afirmó realizar únicamente ejercicio terapéutico para la rehabilitación de su lesión. A partir de las encuestas 
también se pudo determinar que del $100 \%$ de los deportistas que empezaron la rehabilitación para lograr mejorías de su lesión, el 100 \% finalizó con el proceso de recuperación.

Como resultado de la investigación estadística presentada anteriormente, es posible concluir que tanto en las lesiones graves como en las recientes se encontró que son las fracturas, las lesiones que se presentan con mayor frecuencia en los patinadores del Club Tequendama. Además, lo anterior tiene una relación directamente proporcional con los resultados arrojados por las encuestas acerca del tejido más afectado por las lesiones sufridas por los deportistas, ya que fue el tejido óseo el que se vio más afectado; y como se puede observar en el marco de referencia, son las fracturas y las fisuras las lesiones que afectan este tipo de tejido. Por otro lado, se puede determinar que es el traumatismo directo, es decir, por causas claramente definidas como caídas o golpes muy fuertes, el reportado como el mecanismo de lesión más frecuente en los patinadores. Además de lo anterior y para finalizar, con el análisis de los resultados se pudo determinar que son las extremidades inferiores las que presentan mayor número de lesiones en los deportistas que practican el patinaje de carreras.

Se realizaron tablas de contingencia para analizar la relación entre algunas de las variables planteadas y de las que se va a hablar a continuación. Para empezar, se pretendió saber en cuál de las tres categorías (pre juvenil, juvenil o mayores) es la que presenta mayor número de lesiones. Como se puede observar en la tabla 12, es la categoría juvenil (15-16 años) con un total de 10 deportistas, la que cuenta con un mayor número de personas que respondieron que sí han tenido alguna lesión por la práctica del patinaje de carreras. Por otra parte, con 8 deportistas que afirmaron haber sufrido alguna vez alguna lesión deportiva, sigue la categoría de mayores (17 años o más) y por último está la categoría pre juvenil, con solo 3 deportistas que afirmaron haber tenido lesiones por la práctica del patinaje.

Para continuar, se quiso conocer si la duración de la práctica por día guarda alguna relación con la presencia de lesiones en este deporte. En la tabla 13 se puede observar que el mayor número de personas que afirmaron haber padecido una lesión por la práctica del patinaje de carreras, entrenan de 2 a 3 horas en el día. Lo que ayuda a deducir que en este caso en específico, el tiempo de entrenamiento diario no es factor causante en la aparición de lesiones en los patinadores, es decir, que no hay un sobre entrenamiento diario que pudiera influir en la aparición de las lesiones en los patinadores, aspecto que se debe tener muy en cuenta por lo mencionado por Osorio, Clavijo, Arango, Patiño y Gallego (2007), quienes dicen que debe existir una perfecta correlación entre la intensidad y la duración de las cargas del entrenamiento, junto con los periodos adecuados de recuperación para que se disminuyan los niveles de riesgo de aparición de lesiones en deportistas. Otro aspecto que se puede evidenciar que no guarda relación con que los patinadores presenten lesiones deportivas, es la cantidad de sesiones 


\section{Escenarios de la formación investigativa}

que realizan a la semana, ya que los que afirmaron haber tenido lesiones alguna vez, en su gran mayoría respondieron que hacen de 5 a 7 sesiones por semana, es decir, que por lo menos se realiza únicamente una sesión al día, lo que no representa mayor carga de entrenamiento.

Por el contrario y en lo que hace referencia a la frecuencia de la práctica deportiva en la semana, se pudo analizar que esta podría tener algún grado de influencia en la aparición de lesiones en patinadores, ya que al hacer la correlación de las personas que dijeron haber sufrido una lesión con la cantidad de días de entrenamiento a la semana, se pudieron observar datos que decían que el mayor número de personas lesionadas aseguran entrenar siete días a la semana. Con lo anterior se puede llegar a pensar que por el entrenamiento diario hay una falta de periodos de descanso necesarios para la correcta recuperación del deportista y que producen una sobrecarga que incrementa el riesgo de padecer lesiones (Osorio et ál., 2007). Otra información arrojada por las encuestas y que podría ayudar a corroborar lo dicho anteriormente, es lo descrito líneas atrás en los resultados, en las que se describió que según lo dicho por los patinadores, el periodo más frecuente en que se han presentado las lesiones que han tenido, es en el entrenamiento (figura 14).

En lo que respecta a las condiciones de la pista y si estas influyen en la presencia de lesiones en patinadores, se pudo determinar que estas dos variables no guardan ningún tipo de relación, ya que la mayoría de deportistas que cuentan haber sufrido lesiones por el patinaje, aseguran que las pistas en las que realizan sus entrenamientos están en buenas condiciones. Esto, por lo tanto, lleva a deducir que las condiciones de la pista no tienen influencia en que los patinadores sufran lesiones.

Para poder darle respuesta a los objetivos planteados es necesario profundizar en la información obtenida sobre las lesiones recientes y las lesiones graves de las que dieron cuenta los patinadores encuestados. Para empezar, se quiere hablar sobre el análisis hecho a partir de las lesiones recientes reportadas por los deportistas. En cuanto a la relación que se estableció para analizar las variables del tipo de traumatismo con la lesión que presentaron los patinadores, se pudo evidenciar que fue el traumatismo directo el que más se testificó había sido el mecanismo por el que los deportistas obtuvieron su lesión. Como se puede observar en la tabla 14, la fractura es la lesión más frecuente dada por traumatismo directo. Esto es consecuente por lo planteado por McRae y Essen (2010), quienes dicen que las fracturas son secundarias a la aplicación de fuerzas que superan los límites de resistencia del tejido óseo, que para el caso del traumatismo directo y relacionándolo con lo respondido por los deportistas en las preguntas abiertas de las encuestas, estas estarían dadas por caídas y fuertes golpes de la zona afectada contra un objeto resistente, que podría ser el material de la pista, las barandas que la rodean, entre otras cosas. 
Con relación a lo presentado anteriormente es pertinente mencionar que las fracturas fueron las lesiones más presentadas en las extremidades superiores (figura 15). Pero, a partir de lo expuesto previamente en los resultados, donde se dijo que las extremidades del tren inferior fueron las más afectadas por las lesiones recientes sufridas por los patinadores, se ve la necesidad de indagar cuál es la lesión que más afecto a esta zona del cuerpo. A partir del cruce de información, se puede observar en la figura 15 que son cinco las lesiones reportadas como las que afectaron las extremidades inferiores, siendo el esguince como la lesión que más se presentó.

Retomando lo planteado en el marco teórico, en donde se decía que para que una lesión se pudiera clasificar como lesión deportiva, esta tenía que cumplir ciertas características. Una de ellas era que la lesión debía causar una restricción en la participación del deportista durante uno o más días después de sucedida. Esta restricción se supone debería formularse por un profesional de la salud que tenga los conocimientos adecuados para determinar la cantidad y la calidad de esa restricción deportiva. A partir de esto, se pudo observar en los resultados que el $66,7 \%$ de los deportistas afirmo que se les había formulado incapacidad médica, con relación a esto, se hizo un cruce de información para ver si las personas cumplieron debidamente con ese tiempo sugerido y se observó que de las 14 personas a las que les fue formulada una incapacidad médica, solamente 6 de ellas aseguraron haber cumplido con el tiempo de restricción deportiva. En el caso de que se hubieran podido aplicar las entrevistas, estas 8 personas que no completaron el tiempo de la incapacidad, habrían sido candidatas perfectas para la aplicación del instrumento de recolección de información, para conocer más a fondo las razones que influyeron en la decisión de no cumplir con la incapacidad médica formulada.

A partir de lo anterior, se hace necesario entonces, conocer el tiempo que esperaron las otras personas para reanudar con la práctica deportiva. Para ello se buscó hacer una relación del tiempo de incapacidad sugerido contra el tiempo que se esperó para reiniciar la práctica del patinaje. Como se puede observar en la tabla 15, solo hay tres de los ocho casos que justifican la respuesta de los que dijeron no haber cumplido el tiempo de incapacidad. El primer caso es de un deportista que afirmó que se le formuló incapacidad por un tiempo superior a 12 meses y contó que reinició la práctica en un lapso de tiempo de 7 a 11 meses, lo que sugiere que no cumplió restricción. El segundo caso es de otro deportista que afirma que se le formuló un tiempo de incapacidad de 2 a 6 meses y que por el contrario empezó a patinar en un lapso de tiempo de un mes o menos; el último caso corresponde a un deportista que contó que se le formuló un mes o menos de incapacidad pero no cumplió esta, ya que afirmó no haber suspendido la práctica deportiva. Desde este punto de vista, hay una inconsistencia porque faltaría justificar el tiempo que esperaron para reanudar con la práctica, las otras cinco personas que afirmaron no cumplir con el tiempo de incapacidad. La res- 


\section{Escenarios de la formación investigativa}

puesta a esto, puede estar dada a partir de que durante la aplicación de las encuestas, en las preguntas que hacían referencia al tiempo de incapacidad médica y al tiempo que se esperó para reiniciar con la práctica del deporte, no se contaba con rangos de respuestas, sino que estas, para el momento de la aplicación, eran preguntas abiertas. Fue en el momento de ingresar la información al software estadístico, que se tomó la decisión de organizar los datos en rangos para que estos pudieran ser utilizados en las estadísticas de los resultados cuantitativos y no se perdiera la información por ser preguntas abiertas de las que se iba a tener diferentes respuestas. Entonces, a partir de la revisión de las encuestas diligenciadas, se puede exponer un ejemplo en el que el deportista afirma que se le formuló un mes de incapacidad pero que la práctica deportiva la reinició en dos semanas. Esta sería la explicación por la que los tiempos quedaron en un mismo rango y no se relacionaría con la cantidad de personas que dijeron no haber cumplido con la restricción de la práctica del deporte.

Con lo referente a las lesiones más recientes que sufrieron los deportistas del Club Tequendama, también se quiso saber si estos fueron sometidos a algún proceso de rehabilitación deportiva. En los resultados se puede observar que el $57,1 \%$ aseguró haber asistido a sesiones de rehabilitación que les ayudaran con sus lesiones deportivas, y después de realizar una correlación, se observó que dos de esas personas dejaron inconcluso el proceso de rehabilitación, porque aseguraron no finalizar con las terapias. A partir de eso se quiso saber cuáles fueron las lesiones a las que no se les realizó el proceso de rehabilitación completo, entonces se realizó un cruce de información con las lesiones reportadas por los deportistas y se pudo evidenciar que un esguince y una fisura son las dos lesiones con las que no se concluyó el proceso de rehabilitación. Cosa que podría significar que los tejidos no alcanzaron su nivel de cicatrización óptimo, que podría conducir a molestias futuras y por ende al agravamiento de las lesiones.

Por otra parte, gracias a lo reportado por los deportistas del Club Tequendama sobre las lesiones graves sufridas, se evidenció en los resultados que al igual que en las lesiones recientes, el traumatismo directo fue el mecanismo de lesión más frecuente. Asimismo se hizo la triangulación de información y se obtuvo también que por traumatismo directo, las lesiones más frecuentes fueron las fracturas (tabla 16), es decir, que estas fueron lesiones que se presentaron de manera repentina y fueron consecuencia de causas claramente definidas como golpes o caídas. Al mismo tiempo y al igual que en las lesiones recientes, las fracturas fueron más frecuentes en las extremidades superiores. Pero al ser las extremidades inferiores las que más frecuencia de lesiones presentaron, se analizó la información y se determinó que esta zona del cuerpo presentó con mayor frecuencia lesiones del tejido muscular, más específicamente, contracturas. Las contracturas, como se especificó en el marco de referencia, son acortamientos involuntarios de las fibras musculares y que tienen un inicio brusco (Prieto, 1998). Esto, presentaría incongruencia con el mecanismo de lesión que señalaron los deportistas 
por el que obtuvieron la lesión, ya que, como se puede observar en la tabla 16, los deportistas que dijeron sufrir alguna contractura, también dijeron que estás fueron por un traumatismo indirecto, es decir, que fueron ocurriendo y desarrollándose de forma gradual y no por un hecho traumático en particular o con un inicio brusco.

En cuanto a lo relacionado con la incapacidad médica, se cruzó la información que se obtuvo a partir de la pregunta que buscaba averiguar si a los deportistas se les había formulado incapacidad por su lesión grave y si se cumplió con ese tiempo de incapacidad sugerido. Nuevamente se comprueba que un mayor número de personas decidió no cumplir con el tiempo de incapacidad formulado. A partir de esto, se quiso relacionar el tiempo de incapacidad sugerido con el tiempo que se esperó para reiniciar la práctica deportiva, y los resultados arrojaron que uno de los deportistas afirmó que se le formuló una incapacidad de un lapso de 7 a 11 meses pero que reinició la práctica del patinaje en un mes o menos. Otro caso cuenta que al deportista se le formularon de 2 a 6 meses de incapacidad, pero solo detuvo la práctica por un mes o menos; y por último, un deportista que afirmó le habían formulado incapacidad de un mes o menos no suspendió la práctica.

Para concluir con el tema relacionado a las lesiones graves, se hizo un cruce de la información obtenida con las preguntas sobre si los deportistas se sometieron a algún tipo de rehabilitación y si este proceso fue concluido por cada uno de ellos. Se pudo observar que los deportistas que hablaron sobre una lesión grave estuvieron en terapias de rehabilitación para la mejora de su lesión y también, se pudo observar que a diferencia de los resultados de las lesiones recientes, todos ellos finalizaron con el proceso de rehabilitación. 


\section{$\mid$ Conclusiones|}

Después de haber analizado la información, se concluye que el porcentaje de presencia de lesiones en el patinaje de carreras es de un alto nivel. Para la poca población a la que se sometió al estudió, el $80,8 \%$ es un alto porcentaje de personas que afirman haber sufrido algún tipo de lesión por la práctica de este deporte. Por otra parte, se puede afirmar que es un porcentaje bajo $(11,5 \%)$, pero comparando con los resultados obtenidos acerca del número de lesiones en el último año, se ve que hay un 3,8 \% que dice hacer sufrido cinco lesiones o más en un solo año, lo que representaría un aspecto preocupante en lo que a la salud y al rendimiento deportivo se refiere, ya que son muchas lesiones para tan corto tiempo. En lo que se refiere al periodo en que los deportistas afirman ser en el que se presentan con mayor frecuencia las lesiones deportivas, es el periodo de entrenamiento el que obtuvo mayor cantidad de porcentaje. Por otro lado, se pudo observar una relación directa de las respuestas de las lesiones graves y las lesiones recientes, ya que todos los resultados tuvieron respuestas muy similares. En cuanto a la lesión que mayor tuvo presencia, las fracturas, en los dos casos fueron las más predominantes, lo que lleva a concluir y ratificando con los datos obtenidos, que el tejido más afectado por las lesiones en los patinadores es el tejido óseo. Además, con lo relacionado al mecanismo de lesión, el traumatismo directo fue el predominante en los dos casos y la zona del cuerpo que presentó una mayor cantidad de lesiones fue el tren inferior.

Un factor que se evidenció como posible influencia en la aparición de lesiones, es el relacionado con la frecuencia de entrenamiento a la semana. Como se manifestó anteriormente, es de gran importancia que los deportistas cuenten con periodos de descanso en los que se puedan recuperar de manera adecuada.

Otra particularidad de la que se debe estar atento para evitar la aparición de lesiones es el que hace referencia a la completa culminación de las incapacidades y las rehabilitaciones deportivas, así como se vio que algunos de los deportistas no cumplían con los tiempos de incapacidad o simplemente no suspendían la práctica deportiva, se puede llegar a ver cómo las lesiones por la falta de su respectiva 
atención pueden ir agravándose y llevando a peores consecuencias, tanto a nivel deportivo como en la salud.

Es importante entender que como se planteó desde el principio, el nivel de accidentalidad para un deporte como el patinaje de carreras es alto, y por lo tanto es difícil prever el momento en que estos puedan ocurrir, como tampoco se puede saber de las lesiones que estos puedan ocasionar. En estos casos, la mejor forma de actuar es a partir de la prevención, el uso de los implementos de protección se vuelve un factor importante en la prevención de lesiones deportivas, sobre todo para lo que hace referencia a las extremidades superiores, que fueron otra zona del cuerpo afectada por lesiones en el patinaje y sobre todo dadas por golpes y caídas que tal vez con la utilización de los implementos adecuados se habría podido evitar. 


\section{Escenarios de la formación investigativa}

\section{Referencias}

Bahr, R. y Maehlum, S. (2007). Lesiones Deportivas: Diagnóstico, tratamiento y rehabilitación. Madrid: Editorial Médica Panamericana.

Daza, J. (2007). Evaluación Clínico-Funcional del Movimiento Corporal Humano. Bogotá: Editorial Médica Panamericana.

Federación Colombiana de Patinaje. (2005). Patinaje. Comité Internacional de Carreras. Reglas Oficiales. Armenia, Colombia: Editorial Kinesis.

Grelon, B. (1998). Curso de Patinaje Sobre Ruedas. Barcelona: Editorial de Vecchi.

Hinrichs, H. (1995). Lesiones Deportivas. España: Editorial Hispano Europea S.A.

López, J. y López, M. (2008). Fisiología Clínica del Ejercicio. Buenos Aires: Editorial Médica Panamericana.

McRae, R. y Essen, M. (2010). Tratamiento Práctico de Fracturas. España: Editorial Elsevier.

Osorio, J; Clavijo, M; Arango, E; Patiño, S y Gallego, I (2007). Lesiones deportivas (pp. 167-177). Iatreia Vol. 20.

Pfeiffer, R. y Mangus, B. (2007). Las Lesiones Deportivas. Barcelona: Editorial Paidotribo.

Prieto, J. (1998). Exploración del Aparato Locomotor. España: Edit. Universidad de Sevilla.

Romo, O. (2000). Medicina Legal: Elementos de Ciencias Forenses. Chile: Editorial Jurídica de Chile. 